\title{
Imitation of a passive avoidance response in the rat
}

\author{
GAIL B. BUNCH and THOMAS R. ZENTALL \\ University of Kentucky, Lexington, Kentucky 40506
}

\begin{abstract}
Rats learned a candle-flame avoidance task following observation of a demonstrator rat (1) learning to avoid the flame, with flame contacts by the demonstrator visible to the observer (experimental group), (2) learning to avoid the flame, with flame contacts by the demonstrator concealed (nonvisual control group), or (3) unable to make contact with the flame (noncontact control group). Experimental observers learned faster than nonvisual and noncontact controls, and the control groups learned faster than the two avoidance-trained demonstrator groups. The results suggest that facilitated learning by the experimental group was due to true imitation, and not to an increase in fear or general arousal produced by nonspecific demonstrator-provided auditory, olfactory, or visual "alarm signals."
\end{abstract}

Imitative learning by rats has been the subject of sporadic study since early research by Church (1957) and Miller and Dollard (1941) showed that rats could learn to follow another rat to food. More recent research has examined acquisition of a barpress response by rats exposed to a barpressing rat (e.g., Corson, 1967; Gardner \& Engle, 1971; Zentall \& Levine, 1972).

Much of the imitative learning research done with rats has involved appetitive reinforcers. One could argue, however, that any predisposition to imitate the behavior of another animal ought to be at least as strong, or stronger, when the consequences of the behavior produce fear or pain in the animal being observed. In nature, learning through the observation of behavior that is negatively reinforced or punished should have more immediately important consequences for the survival of the observing animal.

One study that has examined learning following observation of the performance of an aversively motivated behavior (Lore, Blanc, \& Suedfeld, 1971) found that rats can learn a passive avoidance response (learning to avoid nose contact with a lit candle) faster after exposure to another rat acquiring the same response than can rats previously exposed to an experimentally naive conspecific. Experimental rats were exposed to demonstrator conspecifics while the demonstrators were learning to avoid nose contact with a lit candle. Control rats were also exposed to a rat and a lit candle for comparable time periods, but the demonstrator did not have access to the flame. Lore et al. reported that, when the observers from both groups were exposed to the candle avoidance task, fewer nose-flame contacts and

This research was supported by Grant MH24092 from the National Institute of Mental Health to the second author. We thank Carrie Griffith for her assistance. Requests for reprints should be sent to Thomas R. Zentall, Department of Psychology, University of Kentucky, Lexington, Kentucky 40506. faster candle avoidance occurred for animals that had been exposed to a conspecific learning the task. Thus, the rats learned a passive avoidance response faster after exposure to another animal acquiring the same response. As Lore et al. noted, a number of mechanisms might have been responsible for the facilitated learning through observation of the demonstrator rat. Possible mechanisms include not only the processing of task-relevant visual cues produced by observation of the demonstrator making contact with the flame (i.e., true imitation), but also the processing of cues that may produce an increase in fear or general arousal level, such as (1) rapid movement by the demonstrator in response to fear or pain, (2) auditory cues emitted by the demonstrator in response to fear or pain, or (3) olfactory cues, such as the smell of singed whiskers or the release of a pheromone by the demonstrator. Lore et al. (1971) speculated that "if olfactory [or other nonspecific motivational] cues are sufficient, then the observer would not have to be in visual contact with the demonstrator" (p. 114).

The present experiment studied the effect of opportunity to observe candle avoidance acquisition on the acquisition of the candle avoidance response and separated the contribution of association-specific visual cues provided by the demonstrator (i.e., imitation) from nonspecific fear or arousal producing cues. The present experiment also examined the effects of the mere presence of a conspecific (i.e., social facilitation) on candle avoidance acquisition by rats.

\section{METHOD}

\section{Subjects}

Seventy-two Long-Evans male rats were reared with littermates in community cages until 33 days old and were then placed in individual cages. The experiment began when the rats were 40 days old. Food and water were continuously available before and after the experimental session. 


\section{Apparatus}

The test apparatus was an outer frame $(21 \mathrm{~cm}$ wide $\times 57 \mathrm{~cm}$ long $\times 19 \mathrm{~cm}$ high) that held two wire home cages $(19.8 \mathrm{~cm}$ wide x $24.5 \mathrm{~cm}$ deep $\times 18.5 \mathrm{~cm}$ high) separated by a narrow compartment $(6.0 \mathrm{~cm}$ wide). The cages were placed in the frame such that the front walls $(1.27-\mathrm{cm}$ square hardware cloth) faced each other. The aversive stimulus was a lit candle $(2.0 \mathrm{~cm}$ in diameter and $6.0 \mathrm{~cm}$ long at the start of each session) inserted in a wooden holder in the floor of the cage. The center of the candle was approximately $2 \mathrm{~cm}$ from the front wall of the cage and equidistant from the side walls. The experiment was conducted in a dimly lit room. Nose-flame contacts were monitored from an adjoining room by means of a video camera and monitor.

\section{Procedure}

Rats were randomly assigned to one of six groups. Each experimental rat $(n=12)$ was transported to the test apparatus in its home cage and was given a 20 -min adaptation period with its home cage placed in one side of the test apparatus. Then, a demonstrator conspecific $(n=12)$, also in its home cage, was placed into the other side of the test apparatus, and a lit candle was inserted into the demonstrator's cage. The demonstrator remained in the cage.until it had successfully avoided the candle for $20 \mathrm{~min}$. Then the demonstrator was removed from its compartment, the demonstrator's candle was removed, and a lit candle was placed in the experimental rat's compartment. The experimental rat's session ended when it successfully avoided the candle flame for $20 \mathrm{~min}$.

Each nonvisual control rat $(n=12)$ was also given a 20 -min adaptation period with its home cage placed in the test apparatus and was then exposed to a demonstrator conspecific $(n=12)$ while the demonstrator was learning to avoid a lit candle; but the demonstrator's candle was concealed from the observer's view by a narrow strip of sheet metal $(6.5 \mathrm{~cm}$ wide $\times 18.5 \mathrm{~cm}$ high $)$ such that the demonstrator's flame contacts were not visible to the observer. (When the experimenter sighted from the outside of either front corner of the observer rat's cage, the candle could not be seen.) For the nonvisual control group, a second lit candle was placed in the center compartment, out of sight and contact of the demonstrator, to control for observation of a lit candle. Once the demonstrator reached criterion, it was removed, both candles were removed, and a lit candle was placed in the nonvisual control rat's cage. The session ended when the nonvisual control rat reached criterion ( 20 min without a candleflame contact).

Each noncontact control rat $(n=12)$ was also given a 20 -min adaptation period and was then exposed to a demonstrator conspecific $(n=12)$. A lit candle was placed in the small compartment between the two wire cages for a period of time equal to the average learning time by the demonstrators for the experimental and nonvisual control groups. Thus, rats in the noncontact control group observed a demonstrator and a lit candle, but the demonstrator could not make contact with the flame. Following the observation period, the demonstrator and the center-compartment candle were removed, and a lit candle was placed in the noncontact control rat's cage. The session ended when the noncontact control rat reached criterion. All three groups of observers were exposed to another rat and a lit candle, but only the experimental group had the opportunity to view the demonstrator actually contact and learn to avoid the lit candle.

\section{RESULTS}

The experimental group made fewer flame contacts than either control group, and the control groups made fewer flame contacts than either demonstrator group. Figure 1 shows the number of rats in each group that

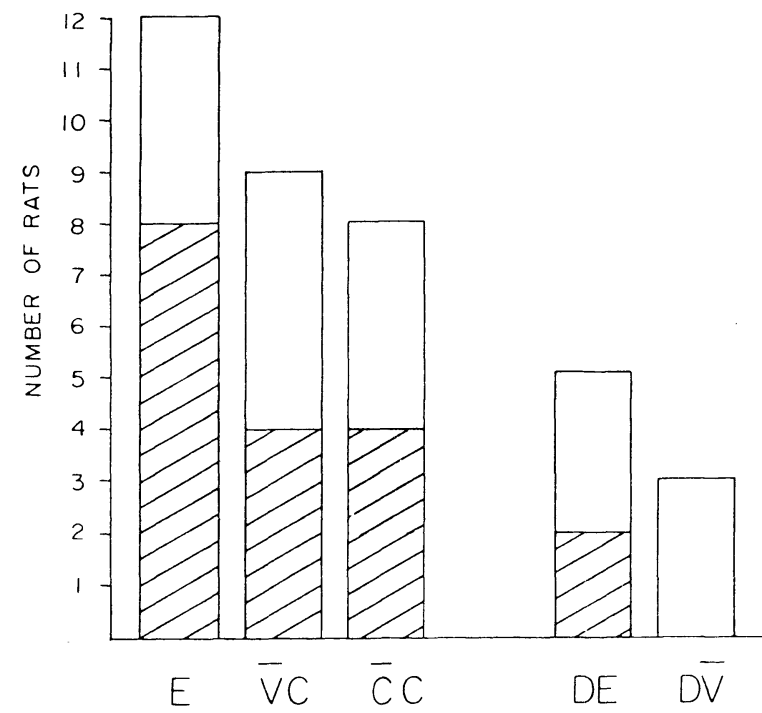

Figure 1. Number of rats that made five or fewer flame contacts to a criterion of 20 min without a contact (combined hatched plus open bars) and number of rats that made one or no flame contacts during the first 5 min (hatched bars) for experimental rats (E) that observed demonstrators (DE) learning not to make contact with the flame, nonvisual control rats $(\overline{\mathbf{V}} \mathrm{C})$ for which observation of flame contact by demonstrators $(D \bar{V})$ was concealed, and noncontact control rats $(\bar{C} C)$ that observed a flame and another rat that made no contacts with the flame.

made five or fewer flame contacts to the criterion of 20 min without a contact.

Because of high within-group variability, it was decided to use a nonparametric statistic, and, due to the small number of subjects in each cell, Fisher's exact test was used in all analyses of the present data. Significantly more rats in the experimental group made five or fewer flame contacts than in the two control groups combined $(p=.04)$. The number of rats that made five or fewer flame contacts in the nonvisual control group was not significantly different from the noncontact control group $(p=.50)$. Finally, the number of rats that made five or fewer flame contacts in the two control groups was significantly different from the two demonstrator groups $(\mathrm{p}=.01)$, although the two demonstrator groups did not differ significantly in that respect $(p=.33)$.

The criterion used in the present study, 20 min without a flame contact, was the criterion used by Lore et al. In an earlier study that also examined flame avoidance in rats, Lore (1969) used fixed 5-min sessions, so the data from the present study were reanalyzed using one or no flame contacts during the first $5 \mathrm{~min}$. Figure 1 also shows the number of rats in each group that made one or no flame contacts during the first $5 \mathrm{~min}$.

The number of rats in the experimental group that made one or no flame contacts in the first 5 min was not quite significantly greater than in the two control groups $(p=.06)$. The number of rats in the nonvisual control group that made one or no flame contacts in the first 
5 min was not significantly greater than in the noncontact control group $(p=.67)$. Finally, the number of rats in the two control groups that made one or no flame contacts in the first $5 \mathrm{~min}$ was significantly greater than in the two demonstrator groups $(p=.04)$, although the two demonstrator groups did not differ significantly in that respect $(p=.24)$. Thus, the two performance measures agree quite well.

\section{DISCUSSION}

In the present study, facilitated acquisition of the passive avoidance response occurred only when the observer was in the presence of a demonstrator making contact with the candle flame. General fear-or arousal-producing visual, olfactory, or auditory cues (or "alarm signals") appear to play little role in facilitated learning. Thus, the present study demonstrated that imitative acquisition of a passive avoidance response in the rat is not produced by nonspecific visual, olfactory, or auditory cues, but rather, by visual cues associated with contacting the aversive stimulus, that is, by true imitation.

The finding that the two control groups acquired the flamecontact avoidance response significantly faster than the demonstrators indicates the detrimental effect of the presence of a conspecific on the learning of such a task. This outcome is consistent with Zajonc's (1965) theory of social facilitation. According to Zajonc the presence of a conspecific will facilitate performance of a dominant or well learned response (see, e.g., Levine \& Zentall, 1974) but will retard the acquisition of, or interfere with, a new or low-probability response (see, e.g., Zentall \& Levine, 1972).

It is possible, however, that relatively poor performance of the demonstrators was related to the fact that the observers were given $20 \mathrm{~min}$ of adaptation to the experimental apparatus and the demonstrators were not. It may be that the differential novelty of the apparatus was responsible for the difference in number of contacts, although one would think that the adaptation period experienced by the observers, if it had any effect, would have allowed for general fear reduction and would have thus tended to increase, rather than decrease, the amount of exploratory behavior shown to the lit candle.

Finally, it should be noted that, while significant social facilitation was found in the present study, Lore et al. (1971) reported only minimally fewer flame contacts by their control group than by their experimental group demonstrators. While the reason for the differential outcome between the two studies is not clear, it may be related to the fact that Lore et al. used older rats (180-190 days old) than were used in the present study.

\section{REFERENCES}

Church, R. M. Two procedures for the establishment of "imitative behavior." Journal of Comparative and Physiological Psychology, 1957, 50, 315-318.

Conson, J. Observational learning of a lever-press response. Psychonomic Science, 1967, 7, 197-198.

Gardner, E. L., \& ENGEL, D. R. Imitational and social facilitatory aspects of observational learning in the laboratory rat. Psychonomic Science, 1971, 25, 5-6.

Levine, J. M., \& Zentall, T. R. Effect of a conspecific's presence on deprived rats' performance: Social facilitation vs. distraction/imitation. Animal Learning \& Behavior, 1974, 2, 119-122.

LORE, R. K. Pain avoidance behavior of rats reared in restricted and enriched environments. Developmental Psychology, 1969, $1,482-484$.

Lore, R. K., Blanc, A., \& Suedfeld, P. Empathetic learning of a passive avoidance response in domesticated Rattus norvegicus. Animal Behavior, 1971, 19, 112-114.

Miller, N. E., \& Dollard, J. Social learning and imitation. New Haven, Conn: Yale University Press, 1941.

Zajonc, R. B. Social facilitation. Science, 1965, 149, 269-274.

Zentall, T. R., \& Levine, J. M. Observational learning and social facilitation in the rat. Science, 1972, 178, 1220-1221.

(Received for publication November 6, 1979.) 